\title{
Hand-Powered Microfluidics for Parallel Droplet Digital Loop-Mediated Isothermal Amplification Assays
}

\author{
Hao Yuan ${ }^{\dagger}$, Jingxuan Tian ${ }^{\S}$, Youchuang Chao ${ }^{\S}$, Yuh-Shiuan Chien", \\ Ren-Hao Luo ${ }^{\ddagger}$ Jun-Yu Guo ${ }^{\ddagger}$, Shanshan $\mathrm{Li}^{\perp}$, Yi-Ju Chou ${ }^{\ddagger}$, \\ Ho Cheung Shum ${ }^{\S *}$, and Chien-Fu Chen ${ }^{\ddagger *}$
}

\footnotetext{
†School of Life Sciences and Engineering, Southwest Jiaotong University, Chengdu, Sichuan 610031, China

Institute of Applied Mechanics, National Taiwan University, Taipei 106, Taiwan

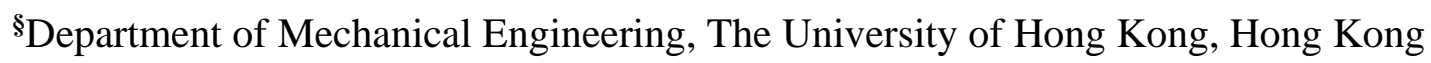
${ }^{\perp}$ Department of Clinical Oncology, The University of Hong Kong-Shenzhen Hospital, Shenzhen, China
}

*Corresponding authors

Ho Cheung Shum (ashum@hku.hk)

Chien-Fu Chen (stevechen@ntu.edu.tw) 


\section{Materials and methods}

1.1 Fabrication process of the Handifluidic Device

The microfluidic device consisted of 2 layers, which were patterned polydimethylsiloxane (PDMS) and PDMS-coated glass. The patterned PDMS layer was fabricated using soft lithography techniques. Specifically, we first designed the pattern using AutoCAD (Auto Desk, USA) as shown in Figure S1. Then photoresist (SU-8 photoresist Microchem, USA) was spin-coated on a silicon wafer at speeds of $1000 \mathrm{rpm}$ to $3000 \mathrm{rpm}$ to achieve channel heights ranging from $25 \mu \mathrm{m}$ to $125 \mu \mathrm{m}$. After UV exposure to crosslink the photoresist and development to wash away the unlinked photoresist, the photoresist-coated silicon wafer was heated to consolidate the photoresist pattern. PDMS base and curing agent (Dow Corning Sygard 184 silicone elastomer, Dow Corning, USA), at a weight ratio of 10:1, were then poured onto the photoresist mold. After degassing and curing at $65{ }^{\circ} \mathrm{C}$ for 2 hours, the PDMS slab was peeled off. Holes (diameter of $1 \mathrm{~mm}$ ) were punched through the PDMS at appropriate points (see Figure S1) using a dermal punch (Miltex Instruments, USA) for loading the LAMP mixture, oil, and attaching the polyethylene tubing ( $\mathrm{PE} / 2$, Scientific Commodities, USA) for connecting the syringe. A glass slide spin-coated with a thin layer of PDMS was used as the bottom layer of the Handifluidic device. Oxygen plasma (PDC-002, Harrick Plasma, USA) was applied to facilitate the bonding of the bottom layer and patterned PDMS.

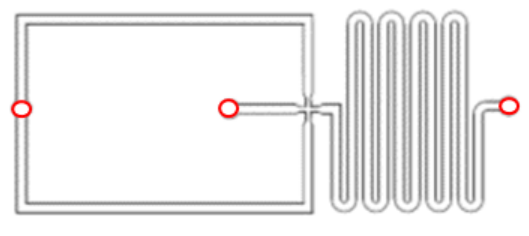

The position needed to be punched with holes

Figure S1. The Handifluidic microchannel pattern, designed using AutoCAD. 


\subsection{Reagents and equipment}

LAMP mixture containing $\left(\mathrm{NH}_{4}\right)_{2} \mathrm{SO}_{4}, \mathrm{KCl}, \mathrm{MgSO}_{4}$, dNTPs, Tween-20, primers, SYTO9, and extracted DNA sample (Double Helix, Guangzhou, China) was used as an inner phase, while mineral oil (Sigma, USA) with 2.5 wt\% ABIL EM 90 surfactant (Evonik Nutrition \& Care GmbH, USA) was used as the outer phase for droplet generation. The DNA of Shigella, Listeria monocytogenes, Bacillus subtilis, and Streptococcus pneumophila were commercially available and bought form Double Helix (Guangzhou, China). Primers used for the detection of these pathogens were proprietary products and were purchased from Double Helix (Guangzhou, China). DNA loading dye and 1kb DNA ladder were purchased from Fermentas (MA, USA), and used for gel electrophoresis. Epoxy (PowerBon, Taiwan) was used to seal the gaps between the needle and syringe in the splitter fabrication process. When not manually actuated, a syringe pump (Cetoni $\mathrm{GmbH}, \mathrm{USA}$ ) was used to control the syringe that was connected to the device to generate droplets with a precise flow rate. A water bath (Troemner, USA) was used to heat the droplets at $63^{\circ} \mathrm{C}$. The droplet generation process was imaged and recorded using a highspeed camera (PCO AG, Germany) connected to an optical microscopy (Olympus, Tokyo, Japan). Fluorescence microscopy (DMIL LED Fluo, Leica Microsystems, Germany) was used to image the fluorescence of the droplets. An open source software - ImageJ (National Institute of Health, USA) — was used to analyze the fluorescence intensity of each droplet. For the experiments of multi-sample processing and calibration curve establishment, $\sim 6500$ droplets were generated for the subsequent analysis. The average volume of the droplet is $\sim 2.3 \mathrm{~nL}$, and the average total volume of the assay is $\sim 15 \mu \mathrm{L}$. 
1.3 The structure of the Handifluidic device

The Handifluidic structure was designed as shown in Figure S2. Specifically, the microfluidic device featured three holes that corresponded to the outer phase inlet for loading the oil/surfactant mixture, the inner phase inlet for loading the LAMP mixture, and the outlet for connecting to the plastic tubing and ultimately to a syringe. The channels in the Handifluidic device consisted of the outer phase channel, inner phase channel, and outlet channel where the oil/surfactant, LAMP mixture, and generated droplets flowed through.

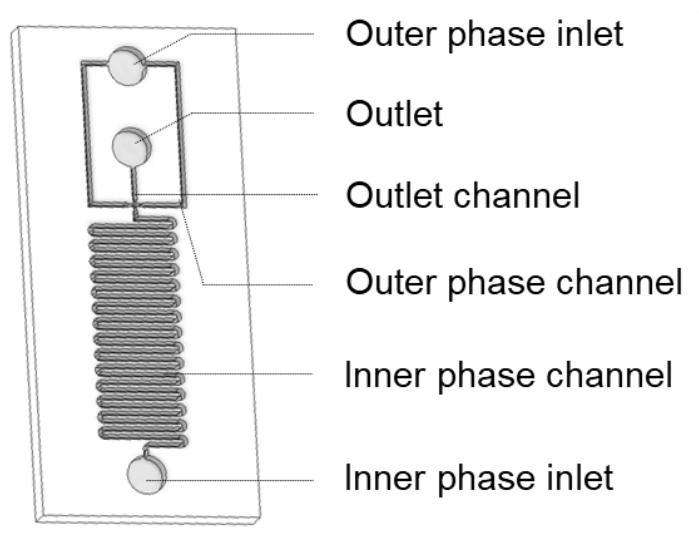

Figure S2. The structure of the proposed droplet generator.

\subsection{Droplet generation process}

To generate the droplets using the Handifluidic device, a $200 \mu \mathrm{L}$ pipette tip containing the LAMP mixture was inserted into the inlet of the inner phase channel, while a $200 \mu \mathrm{L}$ pipette tip containing mineral oil with $2.5 \mathrm{wt} \%$ EM 90 was inserted into the inlet of the outer phase microchannel. The outlet of the device was connected with tubing linked to a droplet container, which was made from a $1 \mathrm{~mL}$ syringe, which was itself connected to another syringe by more tubing, as shown in Figure S3. This second syringe provided the negative- 
pressure-induced flow within the channels. Before droplet generation, the syringe and droplet container were filled with mineral oil mixed with $2.5 \mathrm{wt} \%$ EM 90 to ensure that the negative pressure can be effectively imposed on the Handifluidic device. Applying negative pressure generated by a syringe pump or manual pulling of the syringe draws the LAMP mixture and mineral oil into the microfluidic device, as shown in Figure S3(a). When the two liquids meet within the PDMS-patterned microchannels, droplets are automatically generated and ultimately collected into the bottom of the droplet container, as shown in Figure S3(b).

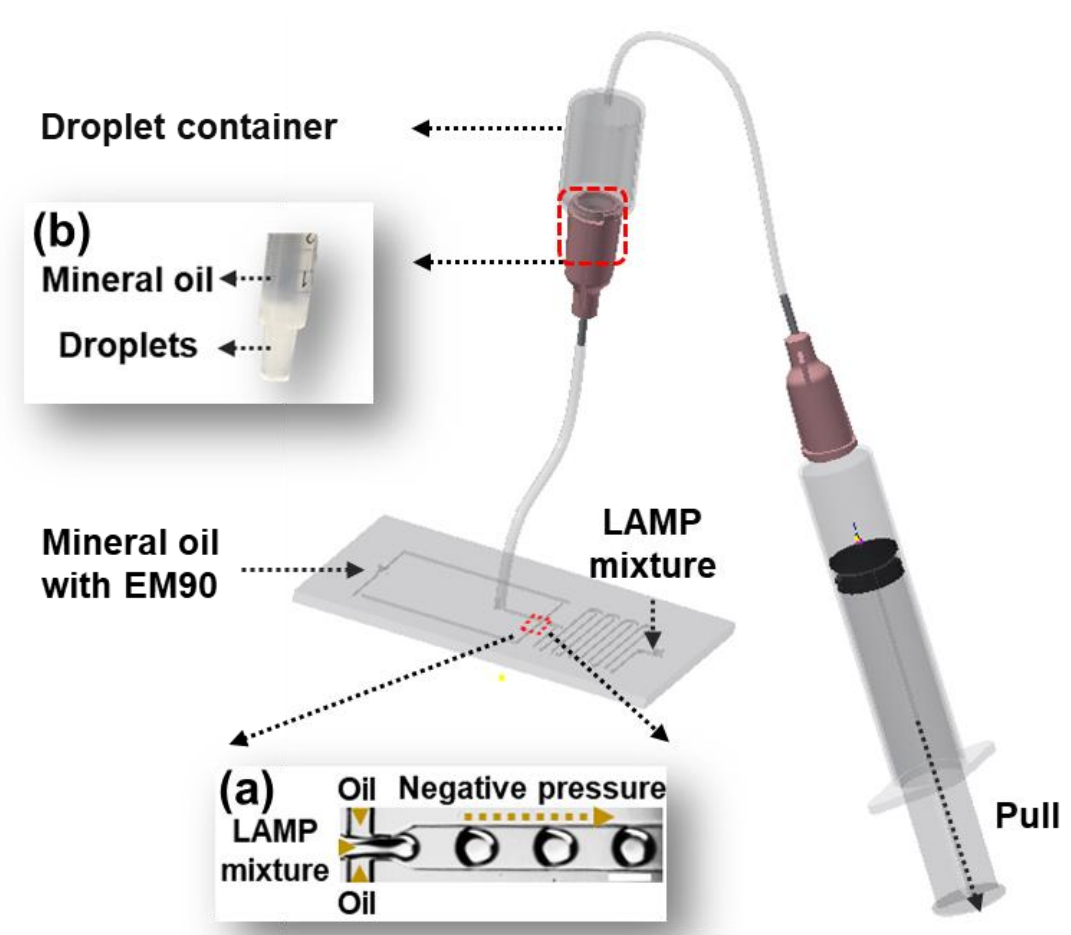

Figure S3. Schematic of the proposed Handifluidic device, droplet container, and the syringe. (a) A microscope image of the droplet generation process in the Handifluidic device. Scale bars are $50 \mu \mathrm{m}$. (b) A photo of the droplet container after collecting the droplets generated in the Handifluidic device. 


\subsection{Droplet heating and imaging}

After emulsifying the LAMP mixture via the Handifluidic device, the resulting droplets were collected in the droplet container. To heat the droplets and conduct the LAMP reaction, the droplet container was disconnected, sealed and heated inside a water bath at $63{ }^{\circ} \mathrm{C}$ for $1 \mathrm{~h}$. After heating, the droplets were removed for analysis by manually compressing the connected syringe to generate a positive pressure, extruding the materials into a 12-well plate for imaging. Due to the density difference between the mineral oil and LAMP buffer, the droplets were precipitated at the bottom of the container, as shown in Figure S3(c), facilitating the droplet extrusion when applying the manual syringe pressure. The 12-well plate was filled with oil before droplet extrusion to prevent coalescence of the droplets in the wells.

To investigate the stability of the droplet, we evaluate the droplet size distribution before and after heating. We found that the droplet size slightly changes from $33.24 \mu \mathrm{m}$ to 34.86 $\mu \mathrm{m}$ as shown in Figure $\mathrm{S} 4$, which may be due to the systematic and random errors during the droplet generation and size measurement, indicating that the stability and uniformity of the droplet can be maintained after heating.

(a) Before heating

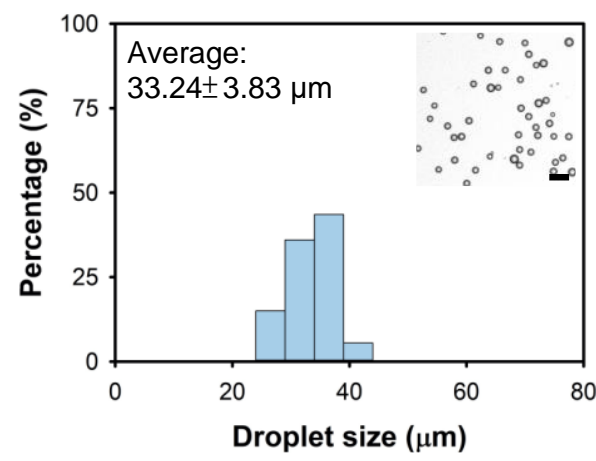

(b) After heating

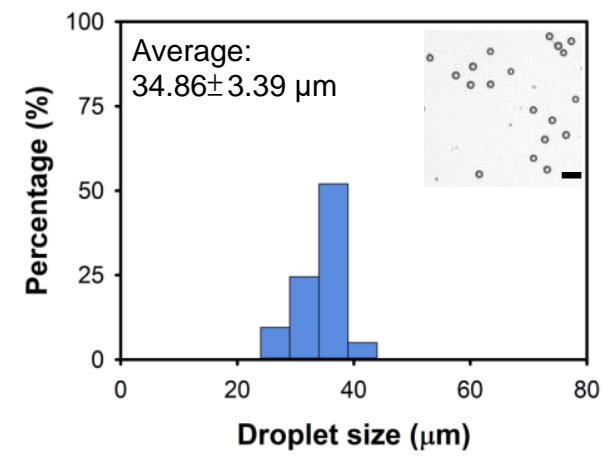

Figure S4. The droplet size distribution before and after droplet heating. The insets show typical microscopic images of the generated droplets. Scale bar is $50 \mu \mathrm{m}$. 


\subsection{Image processing of the ddLAMP assay}

To process the droplet images taken under the fluorescence microscope, we used the analyze particle function in ImageJ software, from which we can determine the fluorescence intensity of each droplet. To indicate the LAMP result, SYTO-9 was added into the LAMP reagent. SYTO-9 refers to a fluorescent intercalating dye bound to the minor groove of double-strand DNA. ${ }^{1}$ It becomes strongly fluorescent with maximal excitation at $485 \mathrm{~nm}$ and emission at $498 \mathrm{~nm}$ (green light). ${ }^{2}$ In this study, the adopted SYTO-9 is commercially available in the LAMP kit. The concentration and the volume added to the LAMP reagent were adjusted by the manufacture to avoid the negative effect of SYTO-9 to the LAMP reaction.

As a result, in a positive ddLAMP assay, DNA amplicons were produced in some of the droplets and bound with SYTO-9, emitting strong fluorescence (i.e., positive droplets); while other droplets produce no amplicons, emitting dim fluorescence (i.e., negative droplets). To avoid miscounting the positive droplets, we set a threshold as 1.5 times of the fluorescence intensity emitting from the negative control, which is commonly used in the existing ddLAMP assay. ${ }^{3,4}$

\section{Accuracy validation of the proposed ddLAMP technique}

To evaluate the accuracy of the ddLAMP technique demonstrated in this work, we prepared a positive and negative test, which were composed of LAMP mixtures containing target DNA and no target DNA, respectively. These mixtures were then emulsified in the proposed Handifluidic device using mineral oil with $2.5 \mathrm{wt} \%$ EM 90 as the outer phase and these LAMP mixtures as the inner phases. After heating the resulting droplets at $63{ }^{\circ} \mathrm{C}$, the 
droplets of the positive and negative tests were separately collected, observed, and imaged by fluorescence microscopy. The amplification results of these tests were indicated by the SYTO-9, which emits strong green fluorescence after binding with DNA. Specifically, in the positive test, amplicons were produced in the droplets containing target DNA and bonded with the SYTO-9 present in the LAMP mixture, emitting strong fluorescence inside the droplets, as shown in Figure S4(a). In the negative test, no amplicons were produced, resulting in dim fluorescence emitted from the droplets, as shown in Figure S4(b).

To confirm the existence and absence of the amplicons in the positive and negative ddLAMP assays, we demulsified the emulsions of the positive and negative assays and extracted the aqueous solutions to run a gel electrophoresis. In addition to the ddLAMP assay, we also performed conventional LAMP assays (independent of droplets) with and without the target DNA and ran gel electrophoresis as control tests. To run the gel electrophoresis, the aqueous solutions extracted from the ddLAMP assays and the solutions after performing conventional LAMP reactions were mixed with DNA loading dye and separated with $2 \%$ agarose gel. A $1 \mathrm{~kb}$ DNA ladder was used as a reference size indicator. After electrophoresis, DNA bands were stained with ethidium bromide and imaged under UV illumination. In our results, we found that typical LAMP bands can be observed, indicating the existence of DNA amplicons in the positive ddLAMP assay, while no DNA amplicons were found in the negative ddLAMP assay, as shown in Figure S4(c). These results were consistent with the conventional LAMP results (Figure S4(c)), indicating that the ddLAMP assays can be performed accurately, without false positive or negative. 

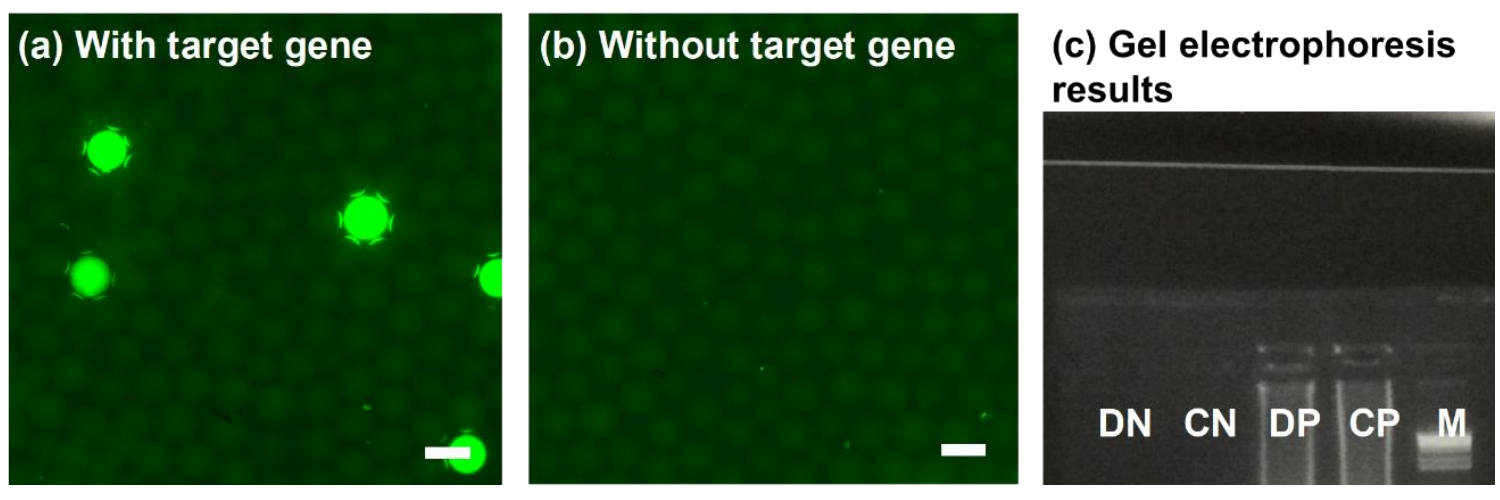

Figure S5. (a, b) Fluorescence microscope images indicating the amplification results of the ddLAMP assay (a) with and (b) without the target DNA. Scale bars are $100 \mu \mathrm{m}$. (c) Gel electrophoresis results of the ddLAMP and conventional LAMP assays. DN: ddLAMP assay containing no target gene. $\mathrm{CN}$ : conventional LAMP assay containing no target gene. DP: ddLAMP assay containing the target DNA. CP: conventional LAMP assay containing the target DNA. M: 1 kb DNA marker.

\section{The relationship between the droplet generation frequency and the suction flow rate}

To investigate the relationship between the droplet generation frequency and suction flow rate of the actuating syringe, a syringe pump was connected to the Handifluidic setup to generate precise flow rates ranging from $300 \mu \mathrm{L} / \mathrm{h}$ to $1000 \mu \mathrm{L} / \mathrm{h}$. After recording the droplet generation process under different flow rates using high speed camera and analyzing the videos, a linear relationship between the droplet generation frequency and suction flow rate can be obtained (Figure S5, blue dots):

$$
\mathrm{Q}=4.1 f-1232.2
$$

where $Q$ is the flow rate and $f$ is the droplet generation frequency, from which an unknown flow rate can be calculated. This relationship excluded the frequency at a flow rate of $1000 \mu \mathrm{L} / \mathrm{h}$ since the video images became blurry at this rate, making it impossible for us to determine the droplet generation frequency. 
Based on equation (1), we can determine the flow rates that are achieved by manually pulling the syringe. Specifically, we recorded the droplet generation process while manually pulling the syringe connected with the Handifluidic device. After analyzing the recorded video, the droplet generation frequency can be observed, from which we can determine the corresponding flow rates (Figure S5, yellow dots) according to equation (1).

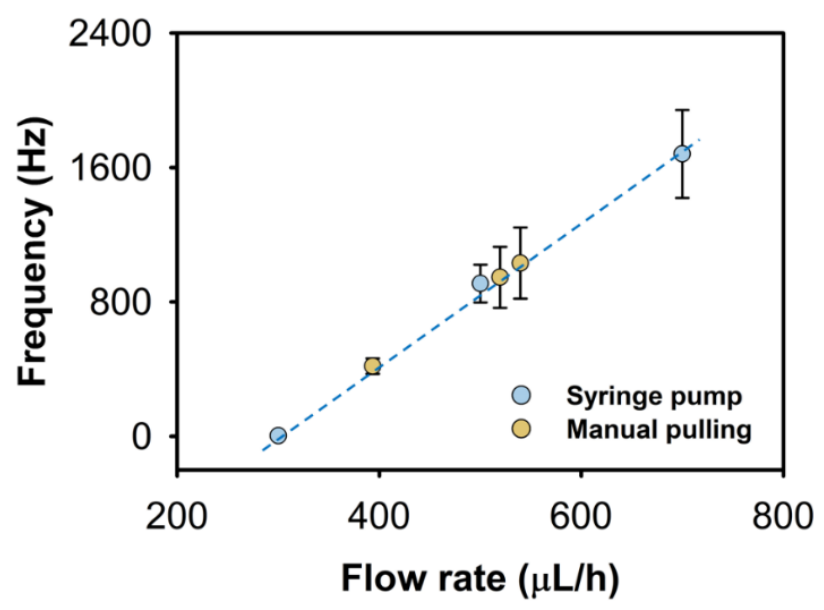

Figure S6. The relationship between the droplet generation frequency and the suction flow rates generated by operating a syringe pump or manually pulling the syringe.

\section{Splitter used for multi-sample processing}

The splitter used for multi-sample processing was fabricated by modifying a syringe, as shown in Figure S6. Specifically, the sides of the syringe were first drilled with 4 holes, which were then connected with tubing using syringe needles, from which it was possible to connect the single syringe to 4 different Handifluidic setups. The gaps between the syringe needles and syringe were sealed using epoxy. The entry point of the syringe where the needle normally connects was also sealed shut to ensure all the negative pressure was generated in the added connections. 


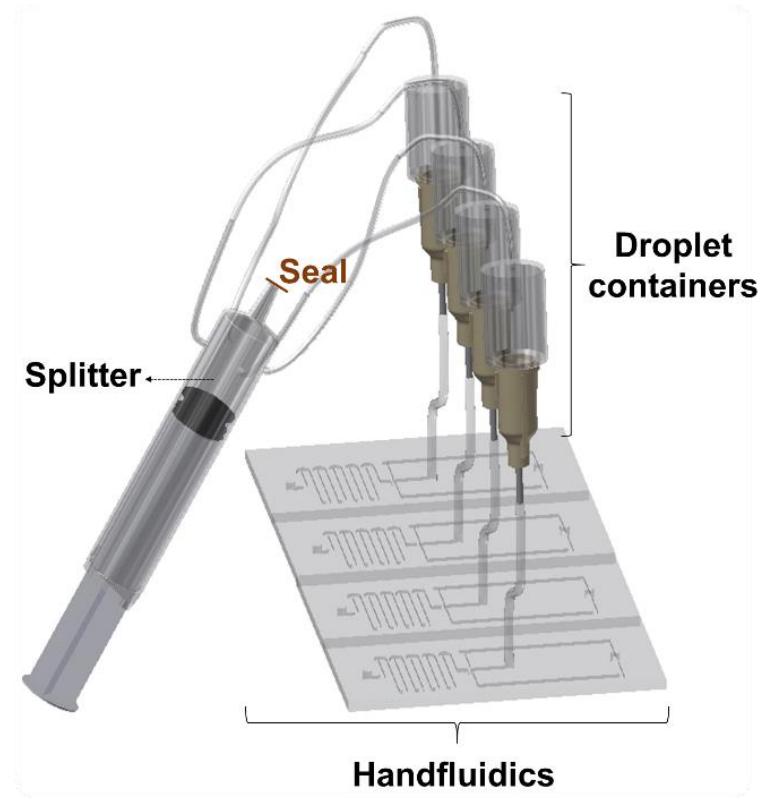

Figure S7. The schematic of the splitter and the connected droplet containers and Handifluidic devices.

\section{The dynamic range of the proposed assay}

Setting confidence level as $95 \%$, the upper limit of the assay with $\sim 6500$ droplet can be calculated as 5 :

$$
\mathrm{M}_{\max }=-\ln \left(1-(1-\alpha)^{\frac{1}{n}}\right)
$$

where $1-\alpha$ is the confidence level, $n$ is number of partitions. Substituting parameters from our experiment into the expression, the upper detection limit of the proposed method can be obtained as:

$$
M_{\max }=-\ln \left(1-0.95^{\frac{1}{6500}}\right)=7.63 \times 10^{4}
$$

To visualize the correspondent distribution, we further used a Monte Carlo-based statistic modeling $^{6}$ and perform a $\sim 6500$-plex 2-D array to simulate the 2-D Poisson process with $7.63 \times 10^{4}$ molecules as follows: 

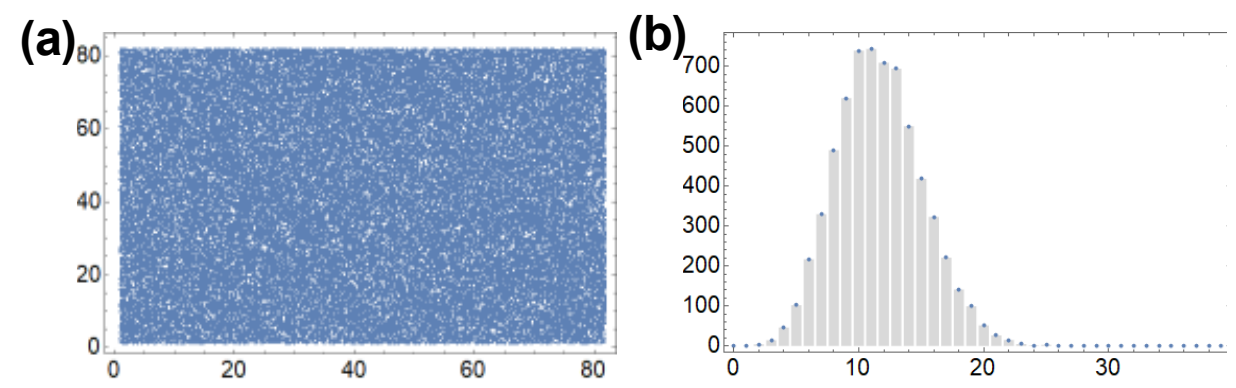

Figure S8. (a) Simulation and (b) statistical analysis of the 2-D Poisson process when the input molecule number is $7.63 \times 10^{4}$. The upper limit of dynamic range could be adjusted to $\sim 10^{5}$ or $\sim 10^{6}$ by adjusting droplets numbers to $\sim 9000$ and $\sim 75000$ respectively.

\section{References}

(1) Quyen, T. L.; Ngo, T. A.; Bang, D. D.; Madsen, M.; Wolff, A. Classification of multiple DNA dyes based on inhibition effects on real-time loop-mediated isothermal amplification (LAMP): prospect for point of care setting. Front. Microbiol. 2019, 10, 2234.

(2) McGoverin, C.; Robertson, J.; Jonmohamadi, Y.; Swift, S.; Vanholsbeeck, F. Species dependence of SYTO-9 staining of bacteria. Front. Microbiol. 2020, 11, 2149.

(3) Rane, T. D.; Chen, L.; Zec, H. C.; Wang, T.-H. Microfluidic continuous flow digital loop-mediated isothermal amplification (LAMP). Lab Chip 2015, 15, 776-782.

(4) Yuan, H.; Chao, Y.; Li, S.; Tang, M. Y. H.; Huang, Y.; Che, Y.; Wong, A. S. T.; Zhang, T.; Shum, H. C. Picoinjection-enabled multitarget loop-mediated isothermal amplification for detection of foodborne pathogens. Anal. Chem. 2018, 90, 13173-13177.

(5) Majumdar, N.; Wessel, T.; Marks, J. Digital PCR modeling for maximal sensitivity, dynamic range and measurement precision. PLoS One 2015, 10, e0118833.

(6) Xia, Y.; Yan, S.; Zhang, X.; Ma, P.; Du, W.; Feng, X.; Liu, B.-F. Monte Carlo modeling-based digital loop-mediated isothermal amplification on a spiral chip for absolute quantification of nucleic acids. Anal. Chem. 2017, 89, 3716-3723. 